\title{
Enfermedad por coronavirus 2019 (COVID-19) y embarazo
}

\section{Coronavirus disease 2019 (COVID-19) and pregnancy}

\author{
Rafael Domínguez Pérez, * Nancy Alva Arroyo, ${ }^{*}$ Juan José Delgadillo Morales, ${ }^{\ddagger}$ Ricardo Enríquez López,\$ \\ Fernando Flores Puente," Esteban Froylan Portillo Urbina," Raúl Chio Magañall
}

Citar como: Domínguez PR, Alva AN, Delgadillo MJJ, Enríquez LR, Flores PF, Portillo UEF et al. Enfermedad por coronavirus 2019 (COVID-19) y embarazo. Acta Med. 2020; 18 (4): 399-406. https://dx.doi.org/10.35366/97267

\section{Resumen}

La enfermedad por coronavirus 2019 (COVID-19) es una enfermedad emergente con un rápido aumento en casos y muertes desde su primera identificación en Wuhan, China en diciembre de 2019. En medio de un brote en rápida evolución que podría tener efectos significativos en nuestra infraestructura médica y de salud pública, deben incluirse las necesidades particulares de las mujeres embarazadas. Se ha descrito que el virus SARSCoV-2 tiene predilección por el receptor ECA II presente en los neumocitos, enterocitos y a nivel placentario. La afección a nivel placentario podría originar hipoperfusión y trombosis, condicionando restricción en el crecimiento intrauterino y/o parto pretérmino. Los síntomas más comunes en la mujer embarazada con COVID-19 son fiebre, tos, dolor muscular, disnea y datos de dificultad respiratoria. El diagnóstico se deberá realizar mediante RT-PCR para SARS-CoV-2 en exudado nasofaríngeo y orofaríngeo. Es recomendable que el personal sanitario que atiende a las gestantes, siga de forma estricta las medidas de prevención recomendadas. Actualmente no existe una vacuna para dicha enfermedad; sin embargo, se han descrito diversos fármacos que podrían ser útiles, tomando en cuenta el riesgo-beneficio de cada uno de éstos.

Palabras clave: Coronavirus, embarazo, COVID-19.

\section{Abstract}

Coronavirus disease 2019 (COVID-19) is an emerging disease with a rapid increase in cases and deaths since its first identification in Wuhan, China, in December 2019. Amid a rapidly evolving outbreak that could have significant effects on our health and medical infrastructure, the particular needs of pregnant women should be included. The SARS-CoV-2 virus has been described as having a predilection for the ACE II receptor present in pneumocytes, enterocytes and at the placental. The placental condition could cause hypoperfusion and thrombosis; restriction on intrauterine growth and/or preterm birth. The most common symptoms in pregnant women with COVID-19 are fever, cough, muscle pain, dyspnoea and respiratory distress data. Diagnosis should be made by rt-PCR for SARS-CoV-2, in nasopharyngeal exudate and oropharyngeal. It is recommended that health workers caring for pregnant women strictly follow the recommended prevention measures. At present, there is no vaccine for this disease, however various drugs have been described that could be useful, taking into account the risk-benefit of each of these.

Keywords: Coronavirus, pregnancy, COVID-19.
* Médico Intensivista adscrito a la Unidad de Cuidados Intensivos del Hospital San Ángel Inn Patriotismo. Ciudad de México. México.

‡ Médico Intensivista. Jefe de la Unidad de Cuidados Intensivos y Coronarios del Hospital San Ángel Inn Patriotismo. Ciudad de México. México.

§ Médico adscrito a la Unidad de Cuidados Intensivos del Hospital Materno Perinatal "Mónica Pretelini Sáenz". Estado de México.

" Médico Cardiólogo adscrito a la Unidad de Cuidados Coronarios del Hospital San Ángel Inn Patriotismo. Ciudad de México. México.

\section{॥ Jefe de la Unidad de Terapia Intensiva del Hospital Ángeles Mocel.} Grupo Ángeles. Ciudad de México.

\section{Correspondencia:}

Rafael Domínguez Pérez

Correo electrónico: rafaeldp@hotmail.com

Aceptado: 02-09-2020. 


\section{INTRODUCCIÓN}

La enfermedad por coronavirus 2019 (COVID-19) es una enfermedad emergente con un rápido aumento en casos y muertes desde su primera identificación en Wuhan, China en diciembre de 2019. Los datos sobre COVID-19 durante el embarazo son limitados; sin embargo, la información sobre enfermedades asociadas con otros coronavirus altamente patógenos (es decir, síndrome respiratorio agudo severo (SARS) y el síndrome respiratorio del medio oriente (MERS) podría proporcionar datos sobre los efectos de COVID-19 durante el embarazo. ${ }^{1}$ Para el SARS, la serie más grande reportó una tasa de letalidad de $25 \%$. Las complicaciones asociadas incluyeron coagulopatía intravascular diseminada, insuficiencia renal, neumonía bacteriana agregada y sepsis. No existe evidencia de transmisión en útero en SARS o MERS. ${ }^{1}$

\section{DEFINICIÓN}

El 30 de enero de 2020, la Organización Mundial de la Salud declaró el brote como una emergencia de salud pública internacional. El 11 de febrero de 2020, la nueva enfermedad por coronavirus (anteriormente conocida como nuevo coronavirus 2019 (2019-nCoV)) recibió el nombre oficial por la Organización Mundial de la Salud de COVID-19. Datos iniciales del brote en China muestran un crecimiento casi exponencial de los casos reportados, confirmando 2.7 millones de casos a nivel mundial el 25 de abril de $2020 .^{2}$

\section{COVID-19 Y EMBARAZO}

Se conoce poco acerca de COVID-19 en el embarazo; sin embargo, se tiene información disponible sobre los efectos del SARS y MERS durante el embarazo y la repercusión fetal.

En medio de un brote en rápida evolución que podría tener efectos significativos en nuestra infraestructura médica y de salud pública, deben incluirse las necesidades particulares de las mujeres embarazadas. ${ }^{2}$

Como en todas las decisiones sobre el tratamiento durante el embarazo, se deben analizar cuidadosamente los beneficios y posibles riesgos de las intervenciones para la madre y el feto. ${ }^{3}$

\section{Susceptibilidad y gravedad de} COVID-19 en el embarazo

Aunque los datos son limitados, se sospecha que las mujeres embarazadas podrían ser más susceptibles a la infección por coronavirus que la población general debido a los cambios inmunológicos durante el embarazo. En una serie de casos de Yang li Lui y colaboradores en Wuhan, China que incluyó 13 pacientes embarazadas, con edad entre 22 y 36 años, se reportó que $23 \%$ presentó complicaciones asociadas a disnea y requirió hospitalización. Sólo una paciente fue ingresada a la unidad de cuidados intensivos por falla orgánica múltiple y síndrome de insuficiencia respiratoria aguda (SIRA) y requirió soporte con membrana de oxigenación extracorpórea (ECMO). El resto de las pacientes cursaron con una enfermedad leve o moderada con recuperación total. ${ }^{4}$

Hasta ahora, en este nuevo brote de coronavirus se ha notado mayor afección al género masculino. Esta diferencia de género podría deberse a diferencias en los informes, susceptibilidad, exposición, reconocimiento y/o diagnóstico de la infección. ${ }^{5}$

\section{FISIOPATOLOGÍA DE LA INFECCIÓN POR SARS-COV-2}

La infección por el virus SARS-CoV-2 se transmite por gotas; al hablar, toser o estornudar se generan partículas que se diseminan de una persona a otra. Si estas gotas quedan en alguna superficie, existe alto riesgo de infección incluso por varios días. Al ingresar el virus al organismo se une al receptor de la membrana de la célula diana y se internaliza a través de la proteína S. El receptor de la célula es el ECA II (enzima convertidora de angiotensina II). Se ha descrito la presencia de dicho receptor en los neumocitos y enterocitos a nivel renal e incluso en la placenta. ${ }^{5}$

La replicación del material genético del virus ocurre en el citoplasma de las células diana mediante endocitosis. La ARN polimerasa sintetiza y decodifica las proteínas $S$, $\mathrm{M}, \mathrm{N}$ y E del virus. Posteriormente mediante exocitosis se liberan copias del virus. ${ }^{5}$

Placenta. Chen S y colaboradores estudiaron en 2020 por primera vez los cambios histopatológicos placentarios en mujeres con embarazo tardío y COVID-19. ${ }^{5}$ El tejido placentario analizado presentó depósitos de fibrina e infartos, lo que podría condicionar hipoperfusión placentaria debido a la presencia de receptores ECA II en la placenta. ${ }^{6}$

Sistema respiratorio. El virus se replica y se genera una respuesta inflamatoria pulmonar, se liberan citocinas proinflamatorias de las células tanto del epitelio respiratorio como endoteliales, provocando vasodilatación, aumento del exudado alveolar e intersticial; el paciente presenta tos seca y disnea secundarias a la dificultad del intercambio gaseoso, situación que condiciona insuficiencia respiratoria aguda hipoxémica (tipo 1). ${ }^{7}$ Cuando los neumocitos tipo I y II se destruyen, el epitelio alveolar inicia una fase de regeneración, sobre todo de los neumocitos tipo II con sus respectivos receptores ECA II, provocando neumonía grave, síndrome respiratorio agudo severo (SARS) y posteriormente COVID-19.8,9 
Sistema cardiovascular. Puede presentarse dolor torácico, arritmias y derrame pericárdico. El endotelio tiene receptores ECA II, cuando el virus se disemina vía hemática causa daño endotelial. ${ }^{10}$ Existe riesgo de insuficiencia venosa profunda y tromboembolia pulmonar. Algunos medicamentos empleados en el momento actual se han asociado con la presencia de arritmias ventriculares. Se han reportado casos de angina e infarto agudo al miocardio tipo 2 asociados a COVID-19. ${ }^{11}$

Sistema hematológico. La inflamación genera fiebre a través de la prostaglandina E2, producción y generación de reactantes de fase aguda como la PCR y ferritina (aumentadas). ${ }^{12}$ Los linfocitos no son infectados, pero sí destruidos, esto condiciona linfopenia. Se ha descrito aumento de DHL (lactato deshidrogenasa) en los pacientes con neumonía por COVID-19. La disfunción endotelial con activación de la cascada de coagulación incrementa el riesgo de trombosis. ${ }^{13}$ Los pacientes con infección por COVID-19 que desarrollan coagulopatía tienen un pronóstico adverso comparado con los que no la desarrollan, por ello el reconocimiento precoz y tratamiento anticoagulante oportuno es necesario. ${ }^{14}$

En los pacientes con infección por COVID-19 que requieren hospitalización, un hallazgo común es el incremento de dímero $\mathrm{D}$ (DD) con niveles más elevados en pacientes de edad avanzada y con comorbilidades, factores que aumentan mortalidad en infección por COVID-19. ${ }^{15}$

Sistema nefrourinario. Mediante la afección del virus a los receptores ECA II a nivel renal, ya sea por vía directa o por mediadores inflamatorios, se origina falla renal aguda con elevación de creatinina, proteinuria, albuminuria y disminución de la tasa de filtrado glomerular.

Sistema digestivo. Una de las manifestaciones clínicas en los pacientes con COVID-19 es la gastroenteritis. Existen receptores ECA II en los enterocitos del intestino delgado que son células diana del virus SARS-CoV-2 condicionando diarrea. ${ }^{15}$

Sistema nervioso. Se ha reportado anosmia en pacientes con COVID-19. Posiblemente al inhalar el virus se tiene contacto con las terminaciones nerviosas del bulbo olfatorio y se genera una respuesta inflamatoria y daño local. Una teoría es que el virus se puede diseminar por vía transneuronal provocando edema cerebral y encefalitis. Existe la probabilidad de que el virus pueda llegar al bulbo raquídeo, generar insuficiencia respiratoria y colapso cardiovascular. ${ }^{16}$

Signos y síntomas de COVID-19 en la mujer embarazada

Datos previos en SARS y MERS sugieren que los hallazgos clínicos durante el embarazo van desde la presentación asintomática hasta la enfermedad grave e incluso en algunos casos defunción. ${ }^{17}$

Los síntomas más comunes en la mujer embarazada con COVID-19 son fiebre (77-90\%), tos (76\%), dolor muscular
Tabla 1: Datos clínicos iniciales en mujeres embarazadas con diagnóstico de COVID-19.

$\begin{array}{lr}\text { Síntomas } & \% \\ \text { Fiebre } & 90 \\ \text { Tos } & 76 \\ \text { Dolor muscular } & 44 \\ \text { Expectoración } & 28 \\ \text { Cefalea } & 8 \\ \text { Diarrea } & 3\end{array}$

Los cambios analíticos iniciales incluyen linfopenia y leucopenia aumento de lactato deshidrogenasa y reacción en cadena de la polimerasa.

Fuente: Protocolo COVID-19 y gestación del Clinic de Barcelona (editado 17-03-2020).

(44\%). Yangli Lui y colaboradores reportaron en un estudio observacional que $85 \%$ de las pacientes con COVID-19 fueron diagnosticadas durante el tercer trimestre del embarazo. La presentación clínica fue similar a la observada en mujeres no embarazadas: fiebre, tos, mialgias, odinofagia y malestar general. Se reportó linfopenia como hallazgo en la biometría hemática. ${ }^{18}$ Todas las pacientes presentaron neumonía, pero ninguna requirió ventilación mecánica y ninguna murió (Tabla 1)..$^{18}$

Yangli Lui y colaboradores reportaron una serie de casos, en la cual $77 \%$ de las pacientes requirieron operación cesárea; la indicación quirúrgica fue en $50 \%$ por indicación materna y fetal. Del total de las pacientes, $46 \%$ presentó parto pretérmino entre las 32-36 semanas de gestación. No existe evidencia actual clínica, serológica ni histopatológica de transmisión vertical de infección por SARS-CoV- $2 .{ }^{19}$

\section{DIAGNÓSTICO}

\section{Procedimientos diagnósticos en los casos sospechosos de COVID-19}

Valoración clínica inicial en área de triage e identificación de caso sospechoso.

Conducir a la paciente con medidas de protección establecidas por cada unidad hospitalaria a la zona de aislamiento establecida de manera protocolaria.

Se deberá realizar muestra de exudado nasofaríngeo y orofaringe para COVID-19 mediante rt- PCR para el virus SARS-CoV-2.

Radiografía de tórax utilizando medidas habituales de protección fetal (delantal abdominal). 
Biometría hemática, tiempos de coagulación, pruebas de función hepática, proteína C reactiva sérica y dímero D. ${ }^{20}$

Monitoreo fetal inmediata: registro cardiotocográfico y ecografía fetal para confirmar viabilidad y bienestar fetal. ${ }^{21}$

\section{Indicaciones de hospitalización de paciente con sospecha de COVID-19}

Inicialmente se considerará como criterio de ingreso hospitalario a toda aquella paciente que cumpla con la definición operacional de caso sospechoso de COVID-19 para estudio de caso y valoración materno-fetal con las medidas de traslado intrahospitalario y protocolo de aislamiento. Las condiciones obstétricas y fetales al momento de la valoración determinarán el destino final de la paciente, en caso de compromiso fetal o materno estará indicada la operación cesárea de acuerdo con los protocolos institucionales. En caso de trabajo de parto en fase activa requerirá vigilancia estrecha y atención de parto vaginal con medidas de aislamiento estricto en relación con los protocolos establecidos en cada unidad hospitalaria. ${ }^{21}$

\section{Datos de gravedad en pacientes embarazadas con sospecha de COVID-19 (Figura 1 y Tabla 2)}

Se considerará paciente con datos de gravedad o en estado crítico aquélla que presente datos francos de dificultad respiratoria (disnea, taquipnea, saturación de oxígeno por oximetría de pulso y/o gasometría arterial menor de $92 \%$ o hipercapnia paCO $\mathrm{CO}_{2}$ mayor de $50 \mathrm{mmHg}$ y/o puntuación en la escala SOFA (Sequencial Organ Failure Assessment) mayor de dos puntos. Esta situación puede ser a su ingreso hospitalario o durante su vigilancia intrahospitalaria, por lo que será de suma importancia su vigilancia estrecha en cada una de las etapas del protocolo de atención. ${ }^{22}$ En caso de paciente en estado crítico o escenario en el que exista un deterioro orgánico materno súbito, estará indicada la interrupción del embarazo vía cesárea, con el fin de mejorar el pronóstico de la madre. ${ }^{22}$

\section{Complicaciones fetales y lactancia materna}

No existe evidencia de transmisión vertical, por lo que es muy poco probable que la infección por COVID-19 pueda producir defectos congénitos. Existen algunas series que han asociado esta patología con aborto espontáneo hasta $2 \%$.

En relación con la restricción del crecimiento intrauterino se ha asociado a SARS-CoV-2 en $10 \%$, esto debido a hipoperfusión placentaria asociada a la presencia de receptores para la enzima convertidora de angiotensina 2 en la placenta. ${ }^{23}$

El parto pretérmino en mujeres con COVID-19 se ha reportado en 39\%, y también se ha asociado previamente con infecciones maternas por SARSCoV y MERS-CoV. ${ }^{23}$

En relación a la lactancia materna no existe ninguna contraindicación para madres con diagnóstico de COVID-19;
Figura 1:

Abordaje inicial en la paciente embarazada con sospecha de COVID-19.

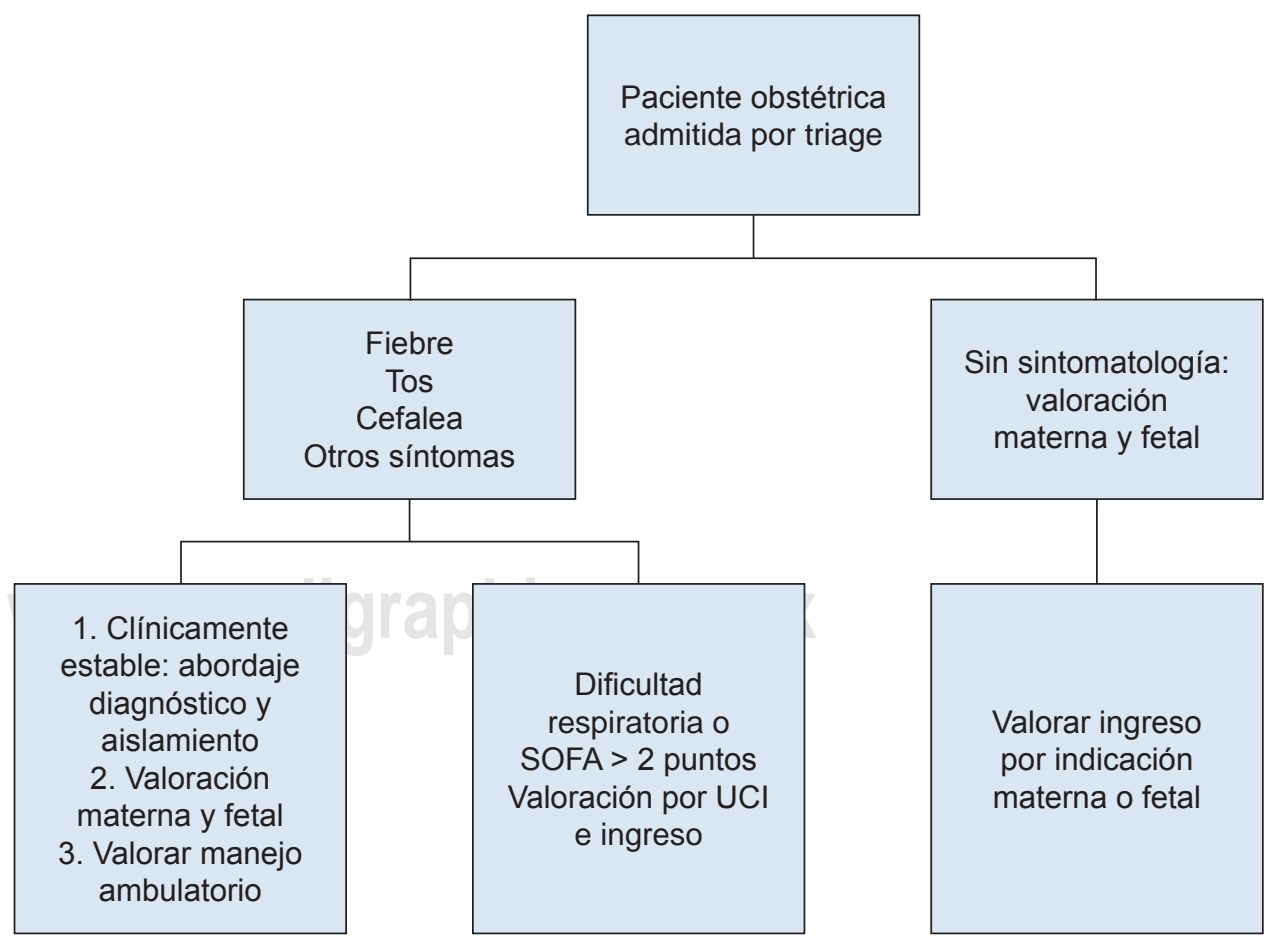


Tabla 2: Criterios de gravedad para pacientes embarazadas con sospecha de COVID-19.

A) Radiografía simple de tórax con infiltrados pulmonares u otras imágenes sugestivas de neumonía

B) Dificultad respiratoria grave:

- Taquipnea o incremento de la frecuencia respiratoria con incapacidad para completar frases

- Uso de la musculatura accesoria (tiraje intercostal, subclavicular o supraclavicular)

- Disnea

C) Saturación de oxígeno por oximetría de pulso $<92 \%$, respirando aire ambiente

D) Agotamiento respiratorio

E) Evidencia de deshidratación severa o estado de choque

F) Alteración del nivel de consciencia: confusión, agitación o convulsiones

G) Cualquier enferma inmunodeprimida grave (trasplantada, infección por el VIH, neutropenia severa)

H) Otros factores considerados por el profesional sanitario responsable del caso: enfermedad rápidamente progresiva o inusualmente prolongada

Fuente: Protocolo COVID-19 y gestación del Clinic de Barcelona (editado 17-03-2020).

sin embargo, se recomiendan medidas estrictas para evitar el contagio por contacto. ${ }^{23}$

\section{Medidas de control de infecciones y pruebas de diagnóstico}

Todas las pacientes, incluidas las mujeres embarazadas, deben ser evaluadas por síntomas de infección respiratoria. Lo ideal es comenzar los procedimientos de detección antes de la llegada del parto. Por ejemplo, al programar citas las pacientes deben recibir instrucciones sobre qué hacer si tienen síntomas respiratorios. ${ }^{24}$

Las pacientes con síntomas respiratorios deben ser separadas de pacientes en espera y se les debe colocar cubrebocas. Pacientes que cumplen con los criterios de caso sospechoso de COVID-19 deben colocarse inmediatamente en aislamiento. ${ }^{24}$

El personal de atención médica debe cumplir con las precauciones estándar para aislamiento por contacto y gotas. Se debe informar de inmediato a epidemiología y a la jurisdicción sanitaria.

\section{Protocolo de atención}

La posibilidad de transmisión vertical es muy poco probable y no se ha podido demostrar en ningún caso durante el brote actual de COVID-19 en China ni en epidemias previas por otros coronavirus similares. Los estudios existentes no han evidenciado presencia del virus en líquido amniótico ni en la leche materna. Los casos descritos de infección en recién nacidos provienen probablemente de transmisión horizontal. ${ }^{25}$

Debido a la elevada contagiosidad de COVID-19 es recomendable que el personal sanitario que atiende a las gestantes siga de forma estricta las medidas de prevención recomendadas (bata, guantes, gorro, gafas y mascarilla según tipo de exposición) y que se proceda a la limpieza del material utilizado (cardiotocógrafos, ecógrafos y resto de material) siguiendo la normativa establecida.

\section{Gestante clínicamente estable sin criterios de ingreso: seguimiento ambulatorio}

Después de la exclusión de criterios de gravedad la paciente podrá ser dada de alta con recomendaciones y medidas de aislamiento domiciliario:

- Reposo domiciliario y control de temperatura, antipiréticos (paracetamol 500 mg vía oral cada ocho horas), hidratación adecuada. Aislamiento domiciliario con medidas de higiene de manos y aislamiento del resto de familiares según el "consejo a la gestante sobre la infección por COVID-19". ${ }^{26}$

Las visitas rutinarias del embarazo, análisis y ecografías de rutina se pospondrán hasta la finalización del periodo de aislamiento. ${ }^{26}$

\section{Paciente con indicación de ingreso por causa obstétrica (Tabla 3)}

Si en una paciente que consulta por motivo obstétrico coinciden síntomas de definición de caso sospechoso, habrá que proceder con las medidas de aislamiento y procedimientos diagnósticos para COVID-19

El personal de medicina materno-fetal no deberá actuar hasta que la paciente esté aislada. No deberá demorarse el procedimiento obstétrico urgente para la obtención 
de las muestras respiratorias y para realizar el protocolo diagnóstico COVID-19. ${ }^{27}$

\section{Maduración pulmonar fetal con esteroides}

La evidencia actual sugiere que en el contexto de una infección COVID-19 la administración de corticosteroides para la maduración pulmonar fetal no produce efectos perjudiciales maternos, por lo que debe individualizarse cada caso y actuar de acuerdo con los protocolos obstétricos de cada unidad médica. ${ }^{28}$

\section{Término de la gestación}

En una gestante con COVID-19 confirmado o caso sospechoso y clínicamente estable no hay indicación de adelantar el parto o realizar una cesárea, excepto al coexistir condiciones críticas que pongan en riesgo la vida de la madre. ${ }^{29}$ El parto vaginal no está contraindicado incluso en la infección activa, ya que no se ha demostrado la transmisión vertical de la enfermedad. ${ }^{29}$

\section{TRATAMIENTO}

\section{Tratamiento médico en mujeres embarazadas con diagnóstico de COVID-19}

La atención clínica de las mujeres embarazadas con COVID-19 se basa en la gravedad de la enfermedad. 85\% de las pacientes embarazadas con COVID-19 presentan sintomatología leve que no justifica estancia intrahospitalaria en ausencia de problemas obstétricos (por ejemplo, parto prematuro) y pueden recibir tratamiento médico ambulatorio. ${ }^{30}$

El tratamiento médico en la pandemia originada por SARS-CoV-2 se encuentra en constante cambio debido

Tabla 3: Medidas inmediatas ante diagnósticos de infección por COVID-19.

- Aislamiento temprano

- Oxigenoterapia. Mantener $\mathrm{SpO}_{2}$ mayor de $92 \%$

- Evitar sobrecarga hídrica

- Tratamiento con antibióticos (en infección bacteriana), SARS-CoV-2 y pruebas de coinfección fetal

- Monitoreo de la actividad uterina y fetal

- Ventilación mecánica temprana en pacientes con falla respiratoria aguda

Fuente: Protocolo COVID-19 y gestación del Clinic de Barcelona (editado 17-03-2020). a la cantidad de estudios que se publican a diario, y a la evidencia científica actual. En esta revisión se menciona el tratamiento propuesto al momento de la elaboración del manuscrito.

\section{Tromboprofilaxis}

Los datos directos sobre el riesgo tromboembólico en COVID-19 sugieren mayor riesgo. ${ }^{31}$ Diversas sociedades internacionales sugieren administrar tromboprofilaxis farmacológica en todas las mujeres embarazadas o en puerperio hospitalizadas con diagnóstico de COVID-19. ${ }^{32}$

La heparina no fraccionada se usa generalmente en mujeres embarazadas que podrían estar próximas al parto porque se revierte más fácilmente que la heparina de bajo peso molecular. La heparina de bajo peso molecular es una opción razonable en mujeres con poca probabilidad de finalizar el embarazo en varios días y en el puerperio. ${ }^{32}$

\section{Esteroides}

Se ha empleado un esquema con dexametasona $(6 \mathrm{mg}$ al día durante 10 días o hasta el egreso) en pacientes no embarazadas gravemente enfermas que reciben oxígeno suplementario o soporte ventilatorio con resultados favorables. ${ }^{33}$ Los glucocorticoides también pueden tener un papel en el tratamiento del choque refractario en pacientes críticos con COVID-19. ${ }^{33}$

En mujeres embarazadas que cumplen con los criterios para el uso de esteroides en el tratamiento materno de COVID-19 y que tienen mayor riesgo de parto prematuro entre 24 y 34 SDG, se sugiere iniciar tratamiento con las dosis habituales de dexametasona (cuatro dosis de $6 \mathrm{mg}$ administradas por vía intramuscular con 12 horas de diferencia) o betametasona (dos dosis de 12 mg administradas por vía intramuscular con 24 horas de diferencia) para inducir la maduración pulmonar fetal, seguida de prednisolona (40 mg al día) o hidrocortisona (80 mg vía intravenosa dos veces al día) para completar el ciclo de esteroides maternos. Esto evita exponer al feto a un curso prolongado de dexametasona o betametasona que atraviesa la placenta en forma metabólicamente activa y puede tener efectos adversos como mayor riesgo de parto prematuro o deterioro del desarrollo neurológico a largo plazo. ${ }^{33}$

\section{Antivirales}

Varios agentes antivirales se están evaluando para el tratamiento de COVID-19. Aunque algunos de estos agentes están clínicamente disponibles para otras indicaciones, su uso en COVID-19 sigue estando bajo investigación. Muy pocos ensayos de medicamentos para el tratamiento de 
COVID-19 incluyen mujeres embarazadas, por ejemplo, los ensayos Solidarity y Recovery. ${ }^{34}$

\section{Remdesivir}

En algunos hospitales, a las mujeres embarazadas con COVID-19 grave se les ofrece remdesivir bajo un protocolo estricto. Remdesivir es un análogo de nucleótido que tiene actividad contra SARS-CoV-2 in vitro y contra los coronavirus relacionados (SARS y MERS-CoV). Se usa sin toxicidad reportada en mujeres embarazadas con enfermedad por el virus del Ébola y Marburg. Se emplea actualmente para tratar pacientes embarazadas con COVID-19 grave. La posología recomendada es de 200 mg como dosis carga (primer día), y dosis de mantenimiento de $100 \mathrm{mg}$ cada 24 horas por nueve días. ${ }^{34}$

\section{Lopinavir-ritonavir}

Lopinavir-ritonavir se usa principalmente para el tratamiento de la infección por VIH, incluso durante el embarazo. Atraviesa la placenta y puede aumentar el riesgo de parto prematuro, pero no se ha observado mayor riesgo de efectos teratogénicos en humanos. Clasificación C durante el embarazo. Su seguridad no está determinada, por lo que su uso se limita de acuerdo al posible beneficio materno. La posología recomendada es de 400 mg/100 mg cada 12 horas por seis a 15 días. ${ }^{35}$

\section{Otros medicamentos}

\section{Tocilizumab}

Es un anticuerpo monoclonal recombinante humanizado al receptor de interleucina 6 (IL-6). Tocilizumab se une específicamente a los receptores de IL-6 (sIL-6R y mIL-6R) tanto solubles como unidos a la membrana, inhibiendo la señalización de la IL-6 mediada a través de estos receptores. ${ }^{35}$ Se ha descrito su utilidad en el tratamiento de la tormenta de citocinas presente en los pacientes con COVID-19. La dosis es de 4 a $8 \mathrm{mg} / \mathrm{kg} /$ dosis, se puede aplicar hasta un total de tres dosis. El tocilizumab se clasifica dentro de la categoría C de riesgo en el embarazo. No existen estudios adecuados ni bien controlados en mujeres embarazadas, por lo que tocilizumab debe utilizarse durante el embarazo sólo si el beneficio justifica el posible riesgo para el feto. ${ }^{35}$

\section{Hidroxicloroquina o cloroquina}

Los datos de los ensayos aleatorios generalmente no sugieren ningún beneficio en la administración de hidroxicloroquina o cloroquina. Además, los efectos maternos adversos incluyen ritmos cardiacos anormales (prolongación del intervalo QT y taquicardia ventricular), especialmente en pacientes que toman otros medicamentos asociados con la prolongación del intervalo QT. La hidroxicloroquina atraviesa la placenta. Se ha observado acumulación en tejido ocular fetal, pero no se ha observado mayor riesgo de anomalías oculares fetales en humanos. Sin embargo, los datos disponibles son limitados y no se puede descartar un riesgo para el feto cuando se usa en diferentes dosis para otras indicaciones. ${ }^{35}$

Los medicamentos en investigación para COVID-19 que se sabe que son teratogénicos incluyen ribavirina y baricitinib.

\section{Plasma convaleciente}

El plasma convaleciente se ha utilizado con éxito en algunas mujeres embarazadas, incluso asociado a remdesivir. Se debe administrar como parte de un ensayo clínico que pueda evaluar la seguridad y la eficacia. En los Estados Unidos, dos ensayos en la Universidad de Pensilvania están estudiando los efectos del plasma convaleciente en pacientes con COVID-19 y están abiertos a mujeres embarazadas que cumplen con los criterios de inclusión. ${ }^{36}$

\section{Vacunación}

Actualmente no existe una vacuna para prevenir $\mathrm{CO}$ VID-19. Desde la publicación de la secuencia genética de SARS-CoV-2 el 10 de enero de 2020, múltiples organizaciones han estado trabajando para desarrollar rápidamente una vacuna para COVID-19. ${ }^{37}$

El desarrollo de esta vacuna se basa y se beneficia del trabajo en las vacunas SARS y MERS. Sin embargo, no se sabe cuándo estará disponible una vacuna segura y efectiva. ${ }^{37}$

\section{CONCLUSIÓN}

En medio de una pandemia originada por un virus letal es importante considerar las condiciones particulares de la población en riesgo. Se conoce cada vez más acerca de COVID y embarazo; sin embargo, existen muchas interrogantes que aún no tienen respuesta. Dentro de los aspectos más importantes está la atención médica que reciben las pacientes embarazadas y las medidas de protección por parte del personal de salud así como los criterios para definir la severidad de la enfermedad, el manejo y tratamiento médico.

\section{REFERENCIAS}

1. Rasmussen S, Smulian J, Lednicky J, Wen T, Jamieson D. Coronavirus disease 2019(COVID-19) and pregnancy: what obstetricians need to know. Am J Obstet Gynecol. 2020; 222: 415-426.

2. López M, Gonce A, Meler E, Plaza A, Hernández S, Martínez PR et al. Coronavirus disease 2019 in pregnancy: A clinical management 
protocol and considerations for practice. Fetal Diagn Ther. 2020; 47: 519-528.

3. Dashraath P, Wong J, Lim M, Lim L, Li S, Biswas A et al. Coronavirus disease 2019 (COVID-19) pandemic and pregnancy. Am J Obstet Gynecol. 2020; 222: 521-531.

4. Ortiz E, Castañeda E, De La Torre A. Coronavirus (COVID-19) infection in pregnancy. Colomb, Medica. 2020, p. 1-7.

5. Alfaraj S, Al-Tawfiq J, Memish Z. Middle east respiratory syndrome coronavirus infection during pregnancy: report of two cases \& review of the literature. J Microbiol Immunol. 2019; 52: 501-503.

6. Gao Q, Chen Y, Fang J. 2019 Novel coronavirus infection and gastrointestinal tract. J Dig Dis. 2020; 21: 125-126.

7. Ye Q, Wang B, Mao J. The pathogenesis and treatment of the cytokine storm in COVID-19. J Infect. 2020; 80: 607-613.

8. Li X, Geng M, Peng Y, Meng L, Lu S. Molecular immune pathogenesis and diagnosis of COVID-19. J Pharm Anal. 2020; 10: 102-108.

9. Wang L, Wang Y, Ye D, Liu Q. Review of the 2019 novel coronavirus (SARS-CoV-2) based on current evidence. Int J Antimicrob Agents. 2020; 55: 10594-10598.

10. Khan S, Jun L, Nawsherwan, Siddique R, Li Y, Han G et al. Association of COVID-19 with pregnancy outcomes in health-care workers and general women. Clin Microbiol Infect. 2020; 26: 788-790.

11. Madjid M, Safavi-Naeini P, Solomon S, Vardeny O. Potential effects of coronaviruses on the cardiovascular system. JAMA Cardiol. 2020; 5: 831-832.

12. Thachil J, Tang N, Gando S, Falanga A, Cattaneo M, Levi M et al. ISTH interim guidance on recognition and management of coagulopathy in COVID-19. J Thromb Haemost. 2020; 18: 1023-1026.

13. Tang N, Li D, Wang X, Sun Z. Abnormal coagulation parameters are associated with poor prognosis in patients with novel coronavirus pneumonia. J Thromb Haemost. 2020; 18: 844-847.

14. Lippi G, Plebani M, Henry B. Thrombocytopenia is associated with severe coronavirus disease 2019 (COVID-19) infections: a metaanalysis. Clin Chim Acta. 2020; 506: 145-148.

15. Levi M, Scully M. How I treat disseminated intravascular coagulation. Blood Adv. 2018; 131: 845-854.

16. Tang N, Bai H, Chen X, Gong J, Li D, Sun Z et al. Anticoagulant treatment is associated with decreased mortality in severe coronavirus disease 2019 patients with coagulopathy. J Thromb Haemost. 2020; 18: 1094-1099.

17. Huang C, Wang Y, Li X, Ren L, Zhao J, Hu Y et al. Clinical features of patients infected with 2019 novel coronavirus in Wuhan, China. Lancet. 2020; 395: 497-506.

18. Taylor F, Toh C, Hoots K, Wada H, Levi M. Towards definition, clinical and laboratory criteria, and a scoring system for disseminated intravascular coagulation. Thromb Haemost. 2001; 86: 1327-1330.

19. Chen N, Zhou M, Dong X, Qu J, Gong F, Han Y et al. Epidemiological and clinical characteristics of 99 cases of 2019 novel coronavirus pneumonia in Wuhan, China: a descriptive study. Lancet. 2020; 395: 507-513.

20. Chen H, Guo J, Wang C, Luo F, Yu X, Zhang W et al. Clinical characteristics and intrauterine vertical transmission potential of
COVID-19 infection in nine pregnant women: a retrospective review of medical records. Lancet. 2020; 395: 809-815.

21. Kong W, Agarwal P. Chest imaging appearance of COVID-19 infection. Radiol Cardiothorac Imaging. 2020; 2: 200028.

22. Liu Y, Chen H, Tang K, Guo Y. Clinical manifestations and outcome of SARS-CoV-2 infection during pregnancy. J Infect. 2020; 28: 1-2.

23. Gao J, Tian Z, Yang X. Breakthrough: chloroquine phosphate has shown apparent efficacy in treatment of COVID-19 associated pneumonia in clinical studies. Biosci Trends. 2020; 14: 72-73.

24. Chu CM, Cheng VC, Hung IF, Wong MM, Chan KH, Chan KS et al. Role of lopinavir/ritonavir in the treatment of SARS: initial virological and clinical findings. Thorax. 2004; 59: 252-256.

25. Della GA, Rizzo R, Pilu G, Simonazzi G. Coronavirus disease 2019 during pregnancy: a systematic review of reported cases. Am J Obstet Gynecol. 2020; 223: 36-41.

26. Tekbali A, Grünebaum A, Saraya A, McCullough L, Bornstein E, Chervenak $F$ et al. Pregnant vs nonpregnant severe acute respiratory syndrome coronavirus 2 and coronavirus disease 2019 hospital admissions: the first 4 weeks in new york. Am J Obstet Gynecol. 2020; 223: 126-127.

27. Chen S, Huang B, Luo DJ, Li X, Yang F, Zhao Y et al. Pregnancy with new coronavirus infection: a clinical characteristics and placental pathological analysis of three cases. Chin Med J. 2020; 49: 5-6.

28. Robertson C, Lowther S, Birch T, Tan C, Sorhage F, Stockman L et al. SARS and pregnancy: a case report. Emerg Infect Dis. 2004; 10: 345-348.

29. Khan S, Jun L, Nawsherwan, Siddique R, Li Y, Han G et al. Association of COVID-19 with pregnancy outcomes in health-care workers and general women. Clin Microbiol Infect. 2020; 26: 788-790.

30. Omer S, Ali S, Babar Z. Preventive measures and management of COVID-19 in pregnancy. Drugs Ther Perspect. 2020; 36: 246-249.

31. Fu B, Xu X, Wei H. Why tocilizumab could be an effective treatment for severe COVID-19? J Transl Med. 2020; 18:1.

32. Thachil J, Tang N, Gando S, Falanga A, Cattaneo M, Levi M et al. ISTH interim guidance on recognition and management of coagulopathy in COVID-19. J Thromb Haemost. 2020; 18: 1023-1026.

33. Horby P, Lim WS, Emberson J, Mafham M, Bell J, Linsell L et al. Dexamethasone in hospitalized patients with COVID-19. Preliminary report. N Engl J Med. 2020: 2-20.

34. Wang M, Cao R, Zhang L, Yang X, Liu J, Xu M et al. Remdesivir and chloroquine effectively inhibit the recently emerged novel coronavirus (2019-nCoV) in vitro. Cell Res. 2020; 30: 269-271.

35. Lacroix I, Bénévent J, Damase MC. Chloroquine and hydroxychloroquine during pregnancy: what do we know? Therapie. 2020; 75: 384-385.

36. Grisolia G, Franchini M, Glingani C, Inglese F, Garuti M, Beccaria M et al. Convalescent plasma for coronavirus disease 2019 in pregnancy: a case report and review. Am J Obstet Gynecol. 2020; 100174.

37. Wu R, Wang L, Kuo H, Shannar A, Peter R, Chou P et al. An update on current therapeutic drugs treating COVID-19. Curr Pharmacol Rep. 2020; 6: 56-70. 